\title{
Web based fingerprint roll call attendance management system
}

Ademola A, Somefun TE, Agbetuyi AF, Olufayo A.

Department of Electrical and Information Engineering, Covenant University, Nigeria

\begin{tabular}{l} 
Article Info \\
\hline Article history: \\
Received Nov 18, 2018 \\
Revised Apr 22, 2019 \\
Accepted May 2, 2019 \\
\hline Keywords: \\
Attendance \\
Authentication \\
Automation \\
Biometric \\
Fingerprint \\
Web technologies \\
\hline
\end{tabular}

\begin{abstract}
Managing people and keeping adequate record of the attendance for most of the tertiary institutions are difficult task. Moreover, the manual taking of attendance adds to the difficulty of this task because it is strenuous, time intensive and can easily be falsified. Meanwhile, With the advent of new technology, a new world of authentication and security has been created via biometrics. This work aims at improving Covenant Universities paper-based attendance by using biometrics, specifically fingerprint technology. It is a web application that works with a fingerprint reader from which the features of the student's fingerprint are extracted during enrolment. This is also needed during the authentication phase which occurs during the roll call attendance in the halls of residence. It is implemented using PHP and MySQL.

Copyright (c) 2019 Institute of Advanced Engineering and Science. All rights reserved.
\end{abstract}

Corresponding Author:

Ademola A,

Department of Electrical and Information Engineering,

Covenant University,

Canaan land, KM 10, Idiroko, Road, P.M.B. 1023, Ota, Ogun State, Nigeria.

Email: ademola.abdulkareem@covenantuniversity.edu.ng

\section{INTRODUCTION}

The use of fingerprint for monitoring attendance feature has been in existence for a while now, with valuable research being done using different methods. Among the methords are the arm 9 based intelligent ststem for biometric fingerprint attandace [1], fingerprint classification using Fuzzy-Neural network method that associate a given finger to one of the existing class [2], fingerprint algorithm method that used for the identification and recongnition of fingerprint [3], classification of parameter of fingerprint based on algorithm tlow method [4] etc. However, with the available variety of methods needed for fingerprint identification and recognition, current techniques have certain drawback in the accuracy and matching speed of the fingerprint mathing algorithm. Although these fingerprint classifications are relevant in many large-scale forensic applications, but are rarely used in biometric authentication [5]. Fingerprint-based identification is the most common and the most used biometric technique. It has been successfully deployed in various sectors and for various applications. For instance, banks have used it for registration and issuing of Bank Verification Numbers (BVN) to their customers; mobile network service providers also use it to register SIM cards for subscribers; it also finds application in security for both residential and commercial buildings. In India and other developed countries, the electronic voting machines based on finger print are being used generaaly for state elections since 1999 up to date [6].

According to (Dhanashree A. G.2011), attendance management system was meant to serve as a substitute for conventional attendance system to lessen the processing time and paper cost [7]. Moreover, attendance is being taken daily at workplaces and in schools in developing and developed countries and this has made attendance recording an interesting research area; This has led to the different development and implementation of the student attendance system using various methods. Attendance capture and recording plays a huge part in the academic success of students. Low attendance in class most times leads to underperformance by students [8,9]. From a survey carried out in New York City state funded schools, 
it was revealed that participation is an important determinant of grades in the school tests. By knowing these realities, attendance of a student ought to be given higher priority irrespective of the school's categories. The increasing number of students in school nowadays makes attendance taking more tedious and therefore traditional method for taking attendance will not be relevant any longer. Producing reports (on daily, weekly or monthly basis) could be very tedious on the part of the teachers. Hence, the need for Automated attendance system in institutions [10].

Several literature discuss different ways of capturing the user's data using fingerprint -based identification but few talks about ways of displaying real-time analysis of data already captured. For instance, in the study of biometric for identifying repeated offenders carried out by Richard Edward [11], there was room for modification of physical appearance and names by the criminals because the work is not displaying real-time analysis of data. This is a great drawback as some inputs is required from the administrator for collating of these results. This is one things this work intends to solve as there would be real-time data analysis of the captured data, hence, reducing the input of the administrator to the barest minimum and making the entire process more automated. The two-tier software framework that was adopted is XAMPP. XAMPP server delivers a windows friendly platform for PHP, Apache web server and MySQL [12].

\section{RESEARCH METHOD}

The system architecture of the roll call attendance management system is a 2-tier architecture. This is because the web application has a user interface that runs on a client with a data layer that gets stored on the server. The two-tier software framework that was adopted in this work is XAMPP server and deliver a windows friendly platform for PHP, Apache web server and MySQL. Figure 1 shows the User interface tier (Client tier) as it communicates with the server tier. Apache which is the web server adopted, stores the web application written in PHP, HTML and CSS. This web application has a user interface which can be run on web browser such as Internet Explorer, Google Chrome or Mozilla. The address typed in by the user on the web browser is converted to the IP address of the web server (Apache- acting the DNS server on the internet) where the database and web application files are stored. Apache facilitates serving the user/client with html content which presents the web application. Apache can only serve dynamic content using server-side scripting language like PHP as in this case. The database stores all information inputted into the system by the users especially the fingerprint in this case, this information is mostly used for matching and authentication.

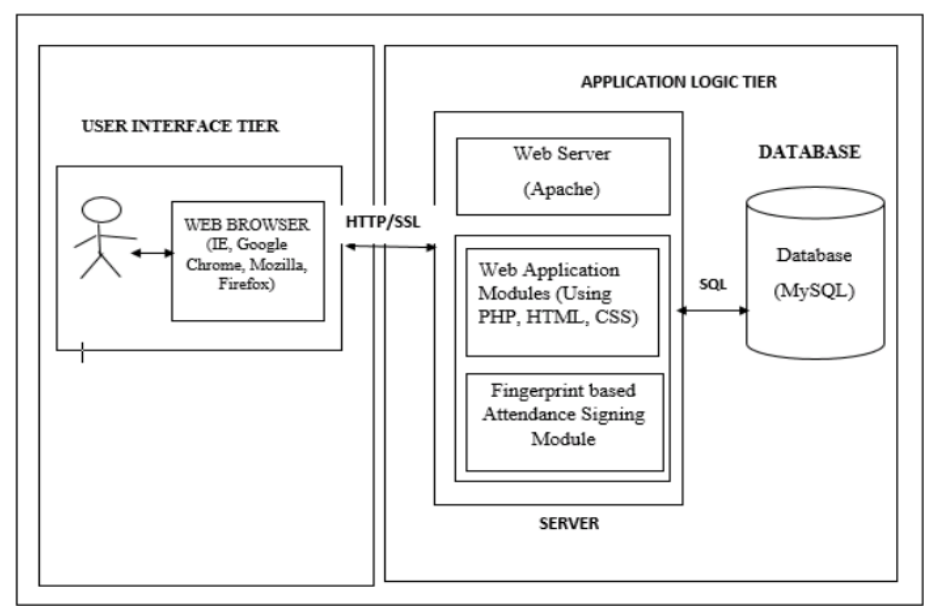

Figure 1. Two-tier architecture of the online biometric based attendance management system

a. User interface tier

The user interface tier is the presentation layer of the web application, it usually runs on web browsers on hardware units like laptops and desktops.

b. Application logic

The application logic is the brain behind the web application as it consists the web server, the server-side scripting in PHP language and the fingerprint-based signing enrolment and authentication module. The fingerprint data stored in the database is hashed to provide an added layer of security. 
The data acquisition involves the fingerprint data acquisition from the students as shown in Figure 2. It occurs during the enrolment phase and authentication phase. The enrolment phase entails the storage of fingerprint data gotten from students as templates in the database. The authentication phase entails the comparing of fingerprint extracted during attendance taking with the template that has been stored in the database. If match is found, then authentication process is completed and result (attendance update) is generated.

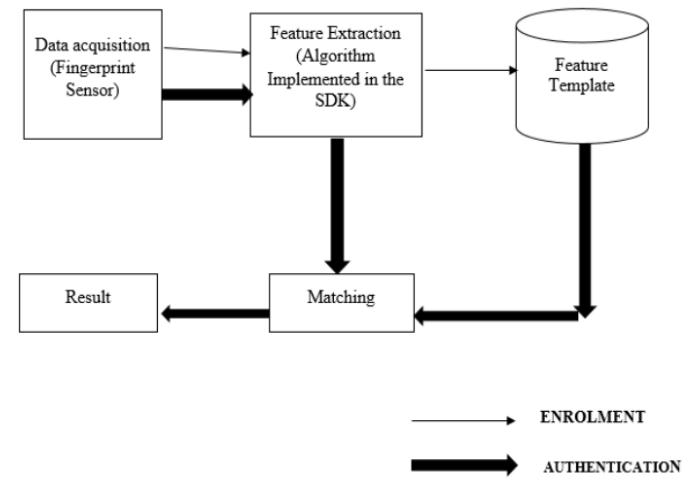

Figure 2. Architecture of the fingerprint biometric attendance signing module

1. Enrolment phase process

The enrolment takes place in the admin module of the system, the processes are shown in Figure 3. The admin adds the student, clicks on the register button for fingerprint extraction process to take place. The student places his/her finger on the sensor which extracts the fingerprint and stores it as a template in the database.

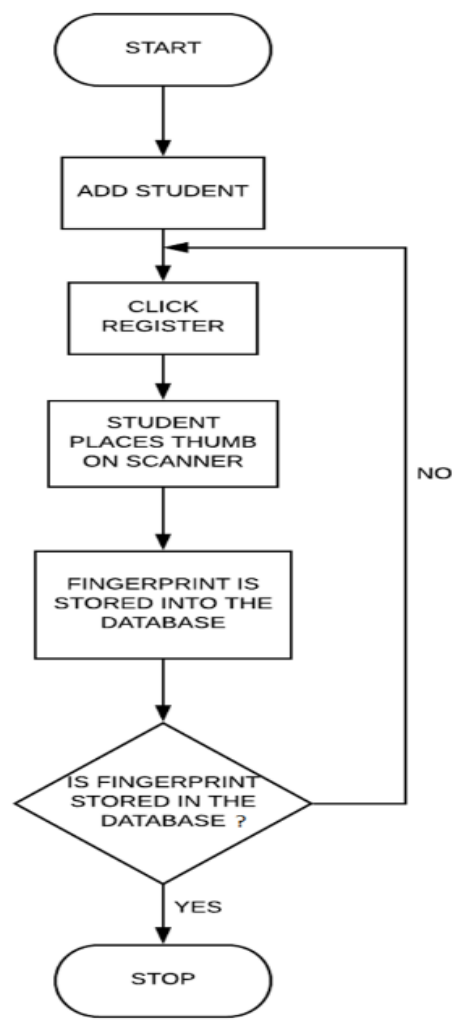

Figure 3. Flowchart representing enrolment process 


\section{Authentication phase process}

The authentication phase process takes place in the lecturer's hall. The student places his/her finger on the sensor, the extracted fingerprint features are then compared with stored fingerprint template to check for a match. Once match is found, the student is marked present as shown in Figure 4.

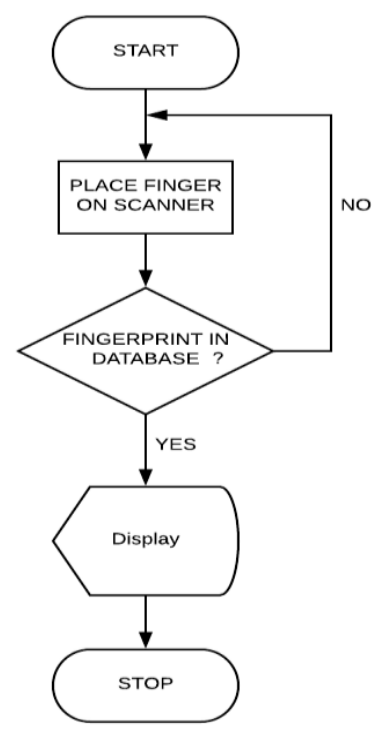

Figure 4. Flowchart representing authentication process

c. Creating a database for fingerprint image

The first step of fingerprint verification is to first create the database. The database is indexed. The XAMPP control panel version 3.2.1 was used to make the computer act as a server. PhpMyAdmin tool was the MySQL database management tool used in this work. There are four tables namely: demo_device, demo_finger, demo_log and demo_user. The fingerprint or thumbprint stored makes it easier for processing as in the case of fingerprint matching. The fingerprint image collected is hashed to add a layer of security to the system by avoiding impersonation. The demo_finger table includes the user_id, finger_id and finger_data. The finger_data is the hashed value of the registered fingerprint. Other requirements of the system are: Flexcode SDK, Local Server (XAMPP server) and Laptop /Personal computer.

Various segments of this work were taken through several tests and verification to ensure the functionality of software application. Figure 5 below shows samples different fingerprints extracted by fingerprint sensor during the enrolment process. These fingerprints are converted into a series of digits and letters that are then stored in the database.

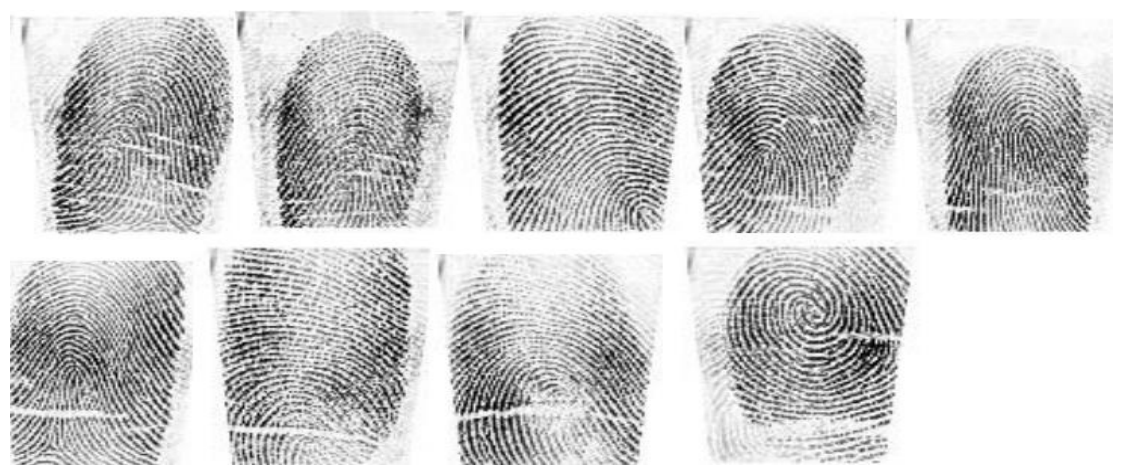

Figure 5. Samples of fingerprint as reflected by fingerprint sensor 
The structural design of the system designed in PHPmyAmin with MySql is depicted in Figure 6. The administrators' login details and students' data are respectively presented in Figures 7 and 8 . The interface for the capturing of roll call attendance is as shown in Figure 9.

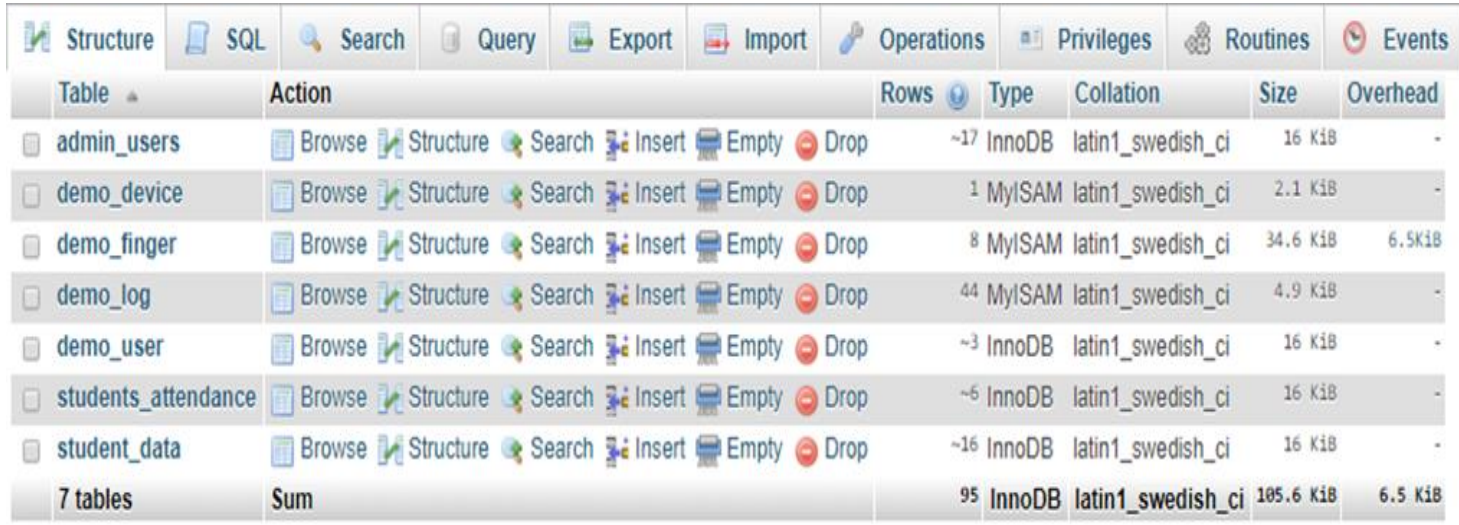

Figure 6. Structure design

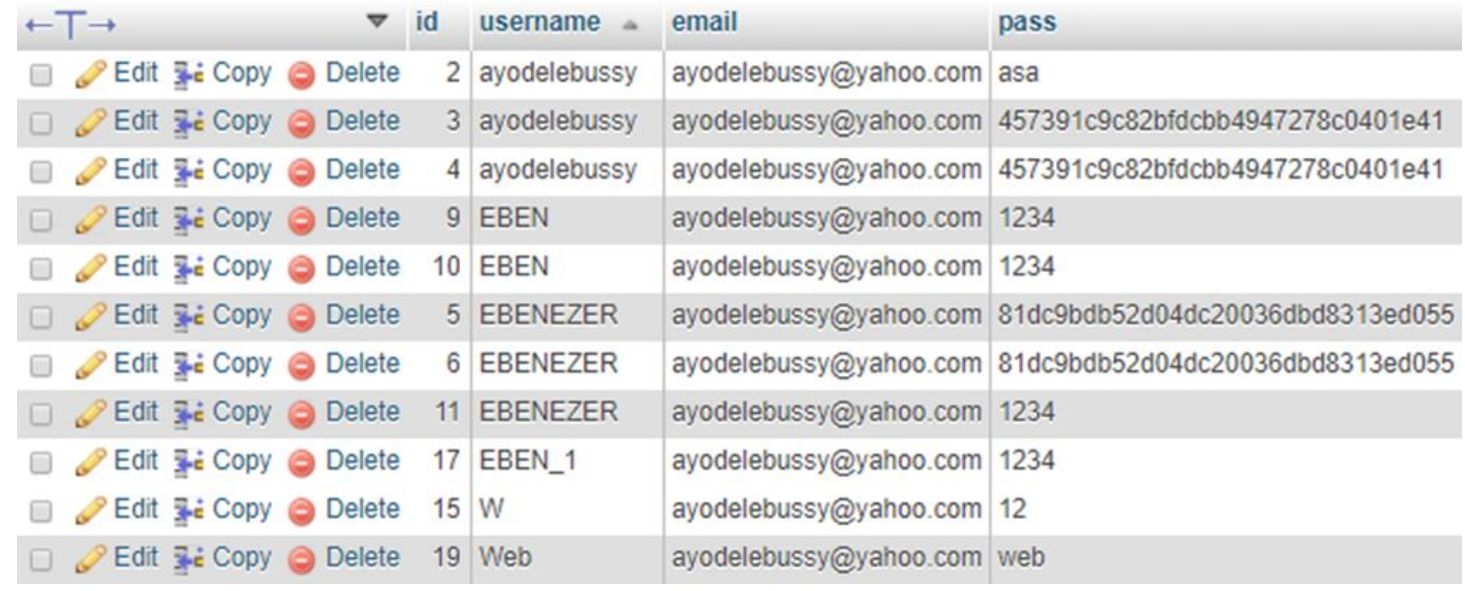

Figure 7. Administrators' login details

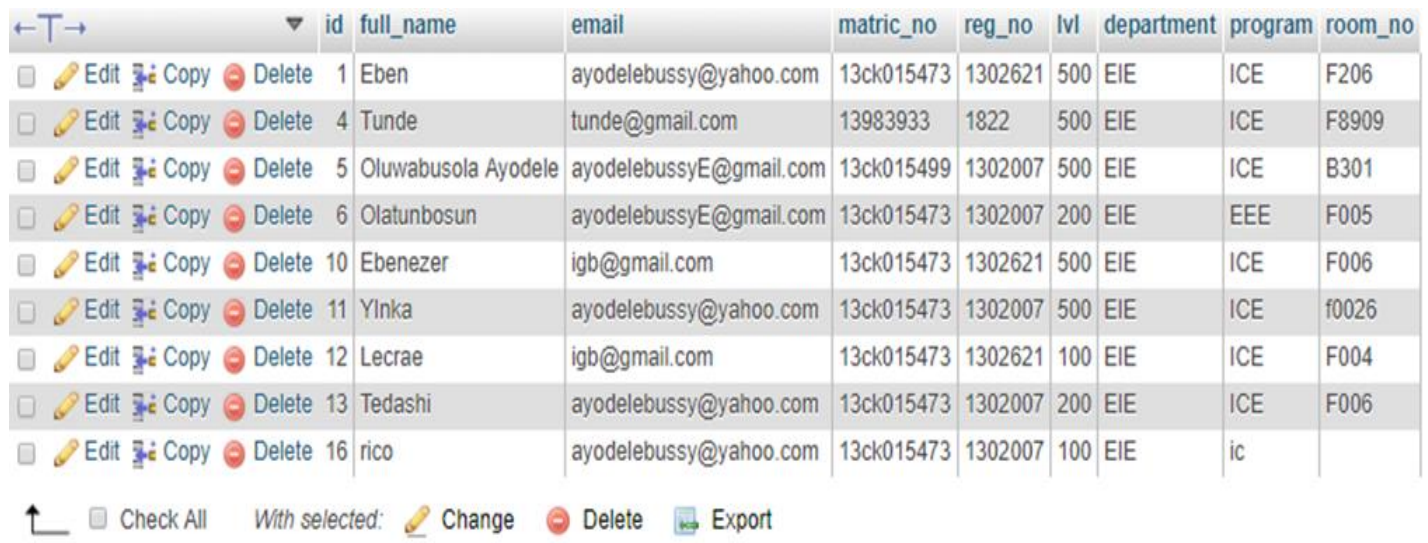

Figure 8. Students' data 


\begin{tabular}{|c|c|c|c|c|c|}
\hline$\leftarrow \mathrm{T} \rightarrow$ & user_id & attendance_status & date_of_capture & day & username \\
\hline 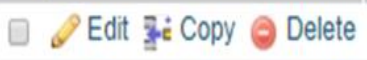 & 17 & & 2018-03-04 08:54:01.000000 & Sunday & Eben4real \\
\hline$\square$ Edit ; $\dot{x}$ Copy $\odot$ Delete & 18 & 1 & 2018-03-04 08:56:26.000000 & Sunday & ArausiD \\
\hline 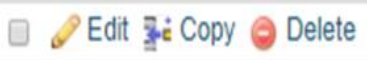 & 19 & 1 & 2018-03-04 20:58:56.000000 & Monday & Eben4real \\
\hline$\square$ Edit $\ddot{\beta} \dot{i}$ Copy $\odot$ Delete & 20 & 1 & 2018-03-04 09:03:47.000000 & Sunday & tedz \\
\hline$\square$ Edit $\underset{\xi}{\mathrm{j}} \mathrm{i}$ Copy $\odot$ Delete & 21 & 1 & 2018-03-13 04:15:04.000000 & Tuesday & Aydoele \\
\hline 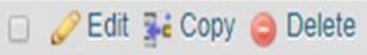 & 22 & 1 & 2018-03-13 04:18:07.000000 & Tuesday & Alabi \\
\hline D Edit ₹్s: Copy $\odot$ Delete & 23 & 1 & 2018-03-14 11:41:29.000000 & Wednesday & Alext \\
\hline
\end{tabular}

Figure 9. Roll call attendance capture details

\section{RESULTS AND ANALYSIS}

The interface for the login module is as given in Figure 10. Both administrator and the students use the same interface. Administrator login to the module to view and analysis the students' attendance frequency on the module, while the students only login to register or sign-in for their courses with already recorded data on database to prevent sign-in error. Any student who does not register for a course would be captured for attendance via the biometric scanner.

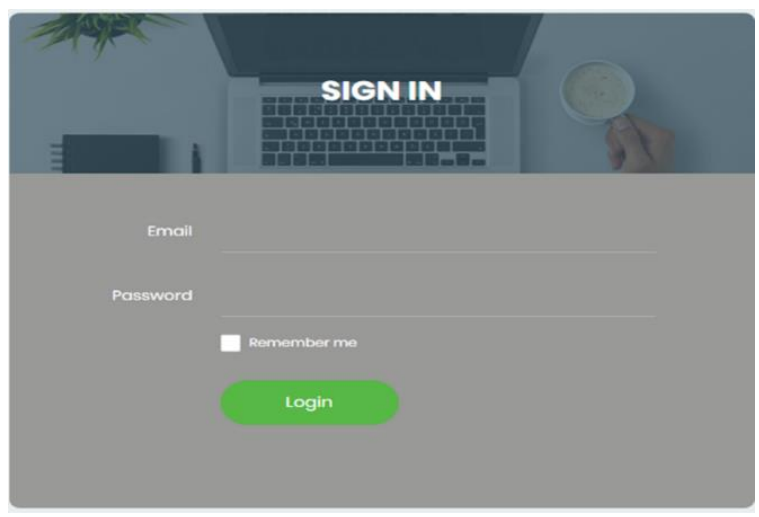

Figure 10. Login module

In order to justify the accuracy of the system, twenty samples were taken, and on each of the samples, 5 signing trials were carried out and the results for each trial was documented in Table 1. Figure 11 shows the plot of the experimental of the fingerprint results.

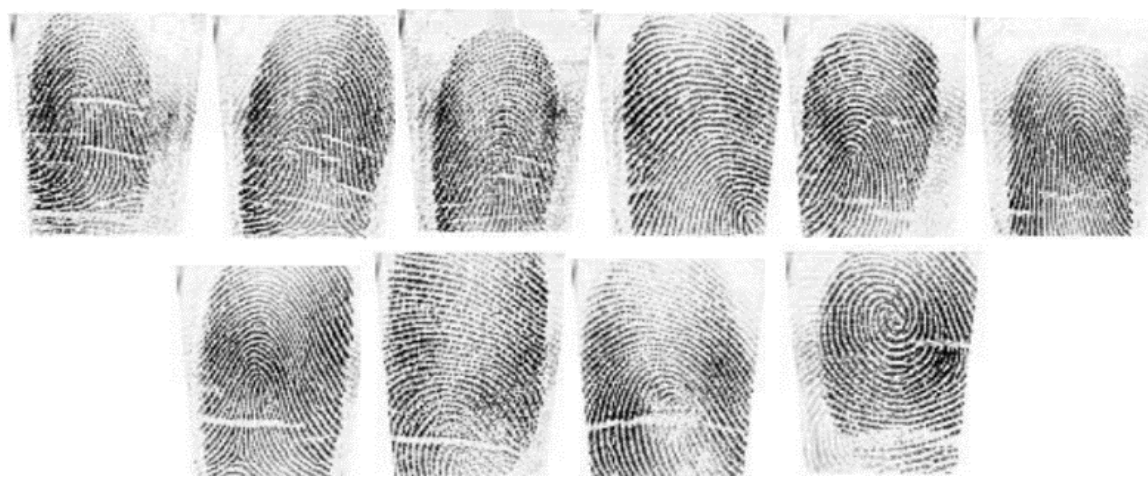

Figure 11. Samples of fingerprint as reflected by the fingerprint sensor 
The False Rejection Rate and False Acceptance Rate are checked in the following manner.

FRR $=$ NFR $/$ NEIA

FAR $=$ NFA / NIIA

Where

FRR $=$ False Rejection Rate

$\mathrm{FAR}=$ False Acceptance Rate

NFR $=$ Number of False Rejection

NEIA = Number of Enrolee Identified Attempt

NFA = Number of False Acceptance

NIIA = Number of Illegal Identified Attempt

Table 1. Result of false rejection rate system test

\begin{tabular}{cccc}
\hline Enrolled Users (sample) & NFR & NEIA & FRR \\
\hline 1 & 1 & 5 & 0.2 \\
2 & 0 & 5 & 0 \\
3 & 1 & 5 & 0.2 \\
4 & 0 & 5 & 0 \\
5 & 0 & 5 & 0 \\
6 & 1 & 5 & 0.2 \\
7 & 1 & 5 & 0.2 \\
8 & 0 & 5 & 0 \\
9 & 2 & 5 & 0.4 \\
10 & 1 & 5 & 0.2 \\
11 & 0 & 2 & 0 \\
12 & 0 & 5 & 0 \\
13 & 1 & 5 & 0.2 \\
14 & 0 & 5 & 0 \\
15 & 2 & 5 & 0.4 \\
16 & 1 & 5 & 0.2 \\
17 & 0 & 5 & 0 \\
18 & 0 & 5 & 0 \\
19 & 1 & 5 & 0.2 \\
\hline
\end{tabular}

Moreever, the administrator can check different chart to summarise the students' frequency in the class as shown in Figures 12 and 13.

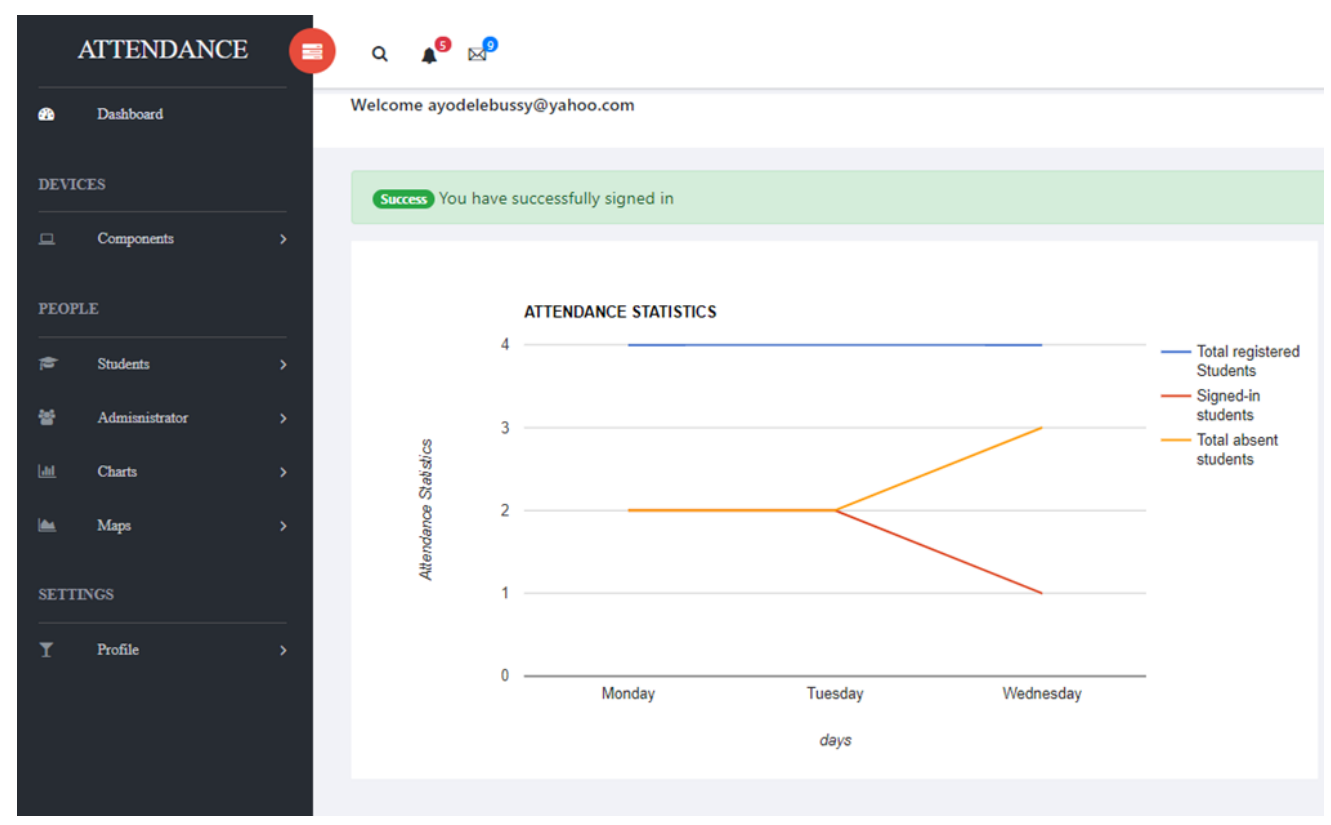

Figure 12. Line chart for attendance captured 


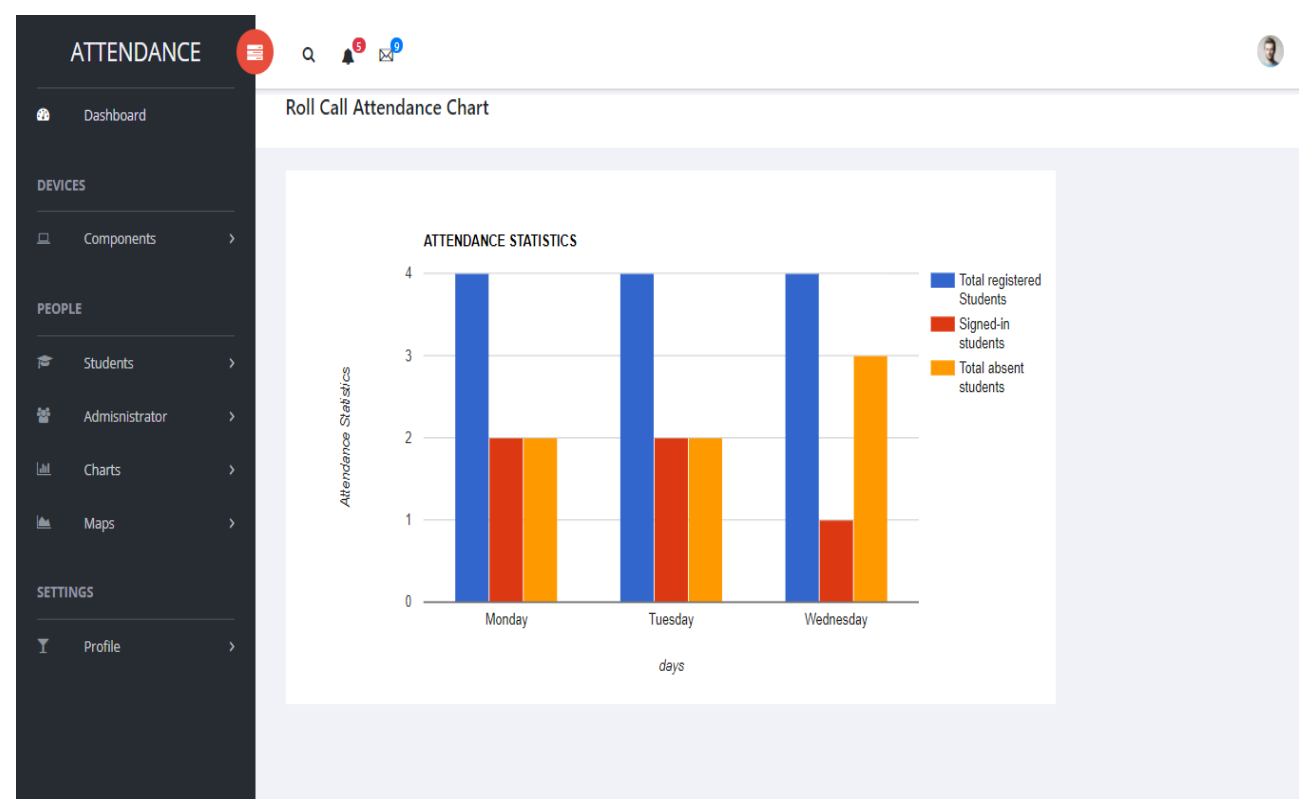

Figure 13. Bar chart for attendance captured

\section{CONCLUSION}

The objective of this work is to develop a fully functional attendance system which is used to carry out a multi-fingerprint operation (registration and verification operations) in real time. The work is embarked with a sole aim of making attendance taking and collation easy and done in real time with immediate update carried out on previously existing data. The purpose of carrying out this work is to help reduce such occurrences of impersonation to the barest minimum. The system would help provide additional security and also prevent unauthorised access of student's data to any individual as fingerprint is unique to every individual.

\section{ACKNOWLEDGEMENTS}

The authors would like to express special thanks to Covenant University for their surport.

\section{REFERENCES}

[1] C. M. Kozierok, The TCP/IP guide: a comprehensive, illustrated Internet protocols reference, No Starch Press, 2005.

[2] S. U. Maheswari and D. E. Chandra, "A review study on fingerprint classification algorithm used for fingerprint identification and recognition," IJCST, vol. 3, pp. 739-45, 2012.

[3] D. Parekh and R. Vig, "Review of Parameters of Fingerprint Classification Methods Based on Algorithmic Flow," in Advances in Computing and Information Technology, ed: Springer, pp. 28-39, 2011.

[4] D. B. V. Raghav and S. K. Bandi, "Digitalized Electronic Voting System," International Journal of Reconfigurable and Embedded Systems (IJRES), vol. 5, pp. 148-152, 2016.

[5] V. K. C. Reddy, B. Sukumar, and S. J. Hussain, "ARM 9 Based Intelligent System for Biometric Figure Authentication," Indonesian Journal of Electrical Engineering and Computer Science (IJEECS), vol. 13, pp. 209-214, 2015.

[6] I. Tazight and M. Fakir, "Fingerprint Classification Using Fuzzy-neural Network and Other Methods," IAES International Journal of Artificial Intelligence (IJ-AI), vol. 3, pp. 129-135, 2014.

[7] L. K. Nam, "Fingerprint Recognition Student Attendance Management System," Universiti Tunku Abdul Rahman, May 2015.

[8] M. Lourde and D. Khosla, "fingerprint identification in biometric system," IJCEE, vol. 2(5), pp. 1793-8163, 2010.

[9] A. Abdulkareem, D. U. Ike, and F. Olowononi, "Development of a Radio Frequency Identification Based Attendance Management Application with A Pictorial Database Framework," International Journal of Research in Information Technology (IJRIT), vol. 2(4), pp. 621-628, Apr 2014.

[10] M. H. Hema Subramaniam, Setyawan Widyarto, "Bar Code Scanner Based Student Attendance System (SAS)." Jurnal TICOM, vol. 1(3), 2013.

[11] H. Colin Beavan, New York City, 2001, Amazon.com. Retrieved 2, "Fingerprints: The Origins of Crime Detection and Murder Case that Launched Forensic Science," August 2014.

[12] S. Y. M. 2012. "Review: XAMPP stacks for Web developers," InfoWorld. Retrieved, 20 January 2015. 